Deformation and orientation during shear and elongation of a polycarbonate/carbon nanotubes composite in the melt

Journal Article

Author(s):

Handge, U. A.; Pötschke, P.

Publication date:

2007-06

Permanent link:

https://doi.org/10.3929/ethz-b-000006552

Rights / license:

In Copyright - Non-Commercial Use Permitted

Originally published in:

Rheologica Acta 46(6), https://doi.org/10.1007/s00397-007-0179-6 


\title{
Deformation and orientation during shear and elongation of a polycarbonate/carbon nanotubes composite in the melt
}

\author{
U. A. Handge · P. Pötschke
}

Received: 24 August 2006 / Accepted: 5 February 2007 / Published online: 4 May 2007

(C) Springer-Verlag 2007

\begin{abstract}
In this study, we focused on the elongational rheology and the morphology of an electrically conductive polycarbonate/multiwalled carbon nanotubes $(2 \mathrm{wt} \%)$ composite in the melt. In shear and melt elongation, the influence of the carbon nanotubes was large when the externally applied stress was small. Consequently, the elastic interactions resulting from the carbon nanotubes dominated in the low frequency range of the shear oscillations. The elongational viscosity of the composite was only moderately influenced by the addition of $2 \mathrm{wt} \%$ carbon nanotubes. Transmission electron microscopy investigations of the stretched composite showed that isolated carbon nanotubes were oriented in elongation. In recovery after melt elongation, the recovered stretch of the composite was much smaller than the recovered stretch of pure polycarbonate. This effect is caused by the carbon nanotubes network, which prohibited large extensions of the macromolecules and led to a yield stress of the composite.
\end{abstract}

Keywords Extensional rheology • Nanocomposites • Carbon nanotubes $\cdot$ Morphology

\footnotetext{
U. A. Handge ( $ه)$

Department of Materials, Institute of Polymers,

ETH Zürich, Wolfgang-Pauli-Strasse 10, HCI H 529, 8093 Zürich, Switzerland

e-mail: ulrich.handge@mat.ethz.ch

P. Pötschke

Department of Polymer Reactions and Blends, Leibniz Institute of Polymer Research Dresden, Hohe Strasse 6, 01069 Dresden, Germany
}

\section{Introduction}

Because of their remarkable mechanical and electrical properties, carbon nanotubes are potential candidates for many applications ranging from electromechanical transducers and scanning probe tips to nanofillers for polymer composites (Dai et al. 1996; Subramoney 1998; Breuer and Sundararaj 2004; Lee et al. 2002; Meincke et al. 2004; Martin et al. 2004; McNally et al. 2005). In composites of polymers and carbon nanotubes, the percolation threshold for electrical conductivity is much lower than in composites with conventional conductive fillers like carbon black (Meincke et al. 2004). In addition, carbon nanotubes can enhance the mechanical performance of polymer composites because of their fibrous shape and their excellent mechanical properties (Coleman et al. 2006). For technological applications, melt processing is one of the most important procedures, and therefore, the melt rheological properties of carbon nanotubes-filled polymers have attracted much attention. Several authors investigated the linear viscoelastic properties of technologically relevant systems in shear and the dependence of the complex modulus on the content of carbon nanotubes (Pötschke et al. 2002, 2004; Mitchell et al. 2002; Seo and Park 2004; Du et al. 2004; Kim and Kim 2006). Huang et al. (2006) studied the influence of nanotube aggregation on the linear viscoelastic properties of a polymer/carbon nanotubes composite. The rheological and electrical percolation thresholds in such nanocomposites were compared by Pötschke et al. (2002, 2004), Meincke et al. (2004), and Zhang et al. (2006b) and were shown to be quite similar.

Fundamentally oriented studies focused on model systems and explored the shear rheology of carbon 
nanotubes suspended in a low viscous polymer melt or in a low molecular solvent in the semi-dilute regime (Hobbie et al. 2003; Hough et al. 2004; Lin-Gibson et al. 2004; Fry et al. 2005; Camponeschi et al. 2006). Hobbie et al. (2003) determined the orientation of carbon nanotubes as a function of the Deborah number in weakly and highly elastic polymer melts during shear using light scattering. The rigidity percolation transition of single wall carbon nanotube suspensions and the interactions between interconnected nanotubes were discussed by Hough et al. (2004). Fry et al. (2005) measured the anisotropy of sheared carbon nanotubes suspensions for various shear rates.

Elongational flows are likewise highly relevant for polymer processing. Typical examples for procedures with a pronounced elongational flow are film and container blowing, melt spinning, and foaming. In addition, anisotropic solid filler particles can be oriented by elongational flows to obtain materials with anisotropic properties (Ooi and Sridhar 2004; Pötschke et al. 2005; $\mathrm{Xu}$ et al. 2005) and flocs of carbon nanotubes can be broken up in elongation (Start et al. 2006).

In this work, we focus on the elongational properties and the morphology of an electrically conductive (and thus technologically highly relevant) composite of polycarbonate (PC) with $2 \mathrm{wt} \%$ multiwalled carbon nanotubes (MWCNT) in the melt. We performed linear viscoelastic shear oscillations and elongational tests with subsequent recovery experiments. The results for the PC/MWCNT ( $2 \mathrm{wt} \%$ ) composite were compared with data of pure PC, and the relation between shear and elongational properties was established. The principal objective of our study was to elucidate the influence of the carbon nanotubes on the elongational viscosity and to investigate the orientation of carbon nanotubes during elongational flows. As melt elongation experiments generally require a much larger amount of material than shear experiments (in our case at least $120 \mathrm{~g}$ of each material), a commercial composite was used in this study.

\section{Experimental}

\section{Materials}

A composite of PC with $2.0 \mathrm{wt} \%$ MWCNT was provided by Hyperion Catalysis International (Cambridge, MA, USA) as granules. The PC/MWCNT (2 wt \%) composite was produced by dilution of a masterbatch of PC containing $15 \mathrm{wt} \%$ MWCNT with a PC that is commercially available from GE Plastics (PC Lexan HF
1110). The MWCNT were produced by chemical vapor deposition (CVD) and had a diameter in the range of $10-15 \mathrm{~nm}$ and consisted of $8-15$ graphite layers that were wrapped around a hollow $5 \mathrm{~nm}$ core (Pötschke et al. 2002). According to the supplier, the length of the MWCNT ranged between 1 and $10 \mu \mathrm{m}$ after production with CVD such that the aspect ratio varied from 1001,000. Table 1 summarizes some physical properties of PC Lexan HF 1110 and of the composite with $2 \mathrm{wt} \%$ MWCNT. A compression-molded sample of this composite with $2 \mathrm{wt} \%$ MWCNT is electrically conductive (Satapathy et al. 2007) with a volume conductivity of $1.6 \times 10^{-2} \mathrm{~S} / \mathrm{cm}$. The high electrical conductivity of this composite indicates a network-like arrangement of the carbon nanotubes.

The volume concentration $\Phi$ of the carbon nanotubes in our composite was approximately $\Phi=1.37 \%$ (Pötschke et al. 2002). The average length $L$ of the nanotubes in the composite roughly equals $L \approx 0.5 \mu \mathrm{m}$ (Lin et al. 2006), which is much smaller than the average length of the nanotubes after production with CVD. This reduction in length is most probably caused by breakage of the nanotubes during the mixing processes. The number $v$ of carbon nanotubes per volume element can be estimated by $v=4 \Phi /\left(\pi L d^{2}\right) \approx 242 \mu \mathrm{m}^{-3}$ with the average diameter $d=12 \mathrm{~nm}$. Hence, $1 / L^{3} \ll$ $1 /\left(d L^{2}\right) \approx v$ holds, and the concentration of carbon nanotubes in this composite belongs to the transition from the semi-dilute to the concentrated regime (Larson 1999) where the interactions between the nanotubes are highly relevant (Schmid et al. 2000; Switzer and Klingenberg 2003) and the free motion of nanotubes is strongly suppressed in contrast to the dilute regime (Trevelyan and Mason 1951).

Granules of PC and the PC/MWCNT (2wt \%) composite were dried at $120^{\circ} \mathrm{C}$ in vacuum for $4 \mathrm{~h}$. Then samples for shear and elongational experiments were prepared by compression molding in vacuum at $240^{\circ} \mathrm{C}$ for $20 \mathrm{~min}$. The diameter of the cylindrical samples for shear experiments was $22.0 \mathrm{~mm}$, and the thickness of these samples was $2.0 \mathrm{~mm}$. The samples for elongational tests were obtained by cutting compression molded plates of dimensions $1.5 \times 40.0 \times 70.0 \mathrm{~mm}^{3}$ into samples of dimensions $1.5 \times 7.0 \times 56.0 \mathrm{~mm}^{3}$. The compression-molded samples were kept in vacuum at room temperature and dried again in vacuum at $120^{\circ} \mathrm{C}$ before the rheological experiments were started.

\section{Rheological measurements}

Linear viscoelastic shear oscillations were performed using the shear rheometer Physica UDS 200 (Physica, Stuttgart, Germany) at $T=190^{\circ} \mathrm{C}$ and $T=210^{\circ} \mathrm{C}$ in 
Table 1 Glass transition temperature $T_{g}$, density $\rho$, and number and weight averaged molecular weights $M_{n}$ and $M_{w}$ of PC (PC Lexan HF 1110) and the PC/MWCNT (2 wt \%) composite using compression molded plates

\begin{tabular}{llllllll}
\hline & $\begin{array}{l}T_{g} \\
\left({ }^{\circ} \mathrm{C}\right)\end{array}$ & $\begin{array}{l}M_{n} \\
(\mathrm{~g} / \mathrm{mol})\end{array}$ & $\begin{array}{l}M_{w} \\
(\mathrm{~g} / \mathrm{mol})\end{array}$ & $M_{w} / M_{n}$ & $\begin{array}{l}\rho \text { at } 25^{\circ} \mathrm{C} \\
\left(\mathrm{g} / \mathrm{cm}^{3}\right)\end{array}$ & $\begin{array}{l}\rho \text { at } 190^{\circ} \mathrm{C} \\
\left(\mathrm{g} / \mathrm{cm}^{3}\right)\end{array}$ & $\begin{array}{l}\rho \text { at } 210^{\circ} \mathrm{C} \\
\left(\mathrm{g} / \mathrm{cm}^{3}\right)\end{array}$ \\
\hline PC Lexan HF 1110 & 147 & 8,700 & 36,200 & 4.16 & 1.1975 & 1.1309 \\
PC/MWCNT (2wt\%) & 146 & 7,300 & 31,000 & 4.25 & 1.2044 & 1.1386 & 1.1185 \\
\hline
\end{tabular}

The molecular weight of PC of the composite was determined by extracting the PC part from the composite following the procedure of Pötschke et al. (2003).

a nitrogen atmosphere. A plate-plate geometry was used with a plate diameter of $25 \mathrm{~mm}$. The gap between the two plates was set to $1.8 \mathrm{~mm}$. The amplitude was $\gamma_{0}=0.02$ resp. $\gamma_{0}=0.03$ such that the deformations did not exceed the linear viscoelastic range. The data of the complex modulus for pure PC were shifted according to the time-temperature superposition principle to the reference temperature $T_{0}=190^{\circ} \mathrm{C}$. For the composite, such a shift procedure was also carried out.

We performed melt elongational experiments using the uniaxial elongational rheometer RME (Meissner and Hostettler 1994). Guidelines for measurements using the RME are reported by Schweizer (2000). The test temperature was $T=190{ }^{\circ} \mathrm{C}$, and the maximum Hencky strain was $\epsilon_{\max }=2.4$. This maximum Hencky strain corresponds to the stretch ratio $\lambda_{\max }=$ $\exp (2.4)=11.0$. Four different Hencky strain rates $\dot{\epsilon}_{0}$ were chosen, i.e., $\dot{\epsilon}_{0}=0.1 \mathrm{~s}^{-1}, 0.3 \mathrm{~s}^{-1}, 0.7 \mathrm{~s}^{-1}$, and $1.0 \mathrm{~s}^{-1}$, such that the macroscopic Hencky strain $\epsilon_{s}=$ $\dot{\epsilon}_{0} t$ increased linearly with time $t$. Tracer particles were spread onto the sample. Then, the true deformation rate of the sample could be determined after the test using a video camera and a particle tracking software. To perform recovery measurements, we installed a pair of scissors into the RME, see also Handge and Pötschke $(2004,2005)$. The scissors were located close to the clamps being opposite to the force measurement system. Immediately after the maximum Hencky strain $\epsilon_{\max }$ had been attained, the scissors cut the sample, which at the same instant of time, was fixed to only one pair of clamps and could recover freely. Using printouts of the video camera images, the distance between selected tracer particles was measured, and the transient recovery was determined during a recovery time of $60 \mathrm{~min}$.

\section{Morphological investigations}

The morphology of selected samples was investigated (1) before elongation, (2) after elongation to the maximum Hencky strain $\epsilon_{\max }$, and (3) at recovery time $t^{\prime}=$ 60 min using transmission electron microscopy (TEM). The samples that were stretched to $\epsilon_{\max }$ were re- moved from the RME using a pair of tweezers and, then, rapidly quenched. For the TEM investigations, the samples were cut along the stretching direction using a Leica Ultracut UCT ultramicrotome (Leica Mikrosysteme, Wien, Austria). The ultramicrotome was equipped with a diamond knife with a cut angle of $35^{\circ}$ (Diatome, Biel, Switzerland) at room temperature. A thickness of $100 \mathrm{~nm}$ of the thin sections was chosen to get a good balance between a high amount of structure elements in the section and a suitable lateral resolution of structural details. The cuts were taken from a middle position within the sample. The TEM used is an EM 912 (LEO, Oberkochen, Germany) operated at $120 \mathrm{kV}$, and the micrographs were taken using an energy filter in zero loss mode for an optimal contrast of the nanotubes.

\section{Results}

Linear viscoelastic shear oscillations

Figure 1 presents the results of the linear viscoelastic shear oscillations for pure PC and the PC/MWCNT ( $2 \mathrm{wt} \%$ ) composite. The dependence of the complex modulus $G^{*}=G^{\prime}+i G^{\prime \prime}$ as a function of $\omega$ at reference temperature $T_{0}=190{ }^{\circ} \mathrm{C}$ is shown in Fig. 1a. The storage modulus $G^{\prime}$ and the loss modulus $G^{\prime \prime}$ of pure PC display the typical behavior of melts of homopolymers: The storage modulus $G^{\prime}$ exceeds the loss modulus $G^{\prime \prime}$ in the plateau at large frequencies. In the intermediate frequency range, we observe the transition from the plateau to the terminal zone. At low $\omega$ values, $G^{\prime \prime}$ is larger than $G^{\prime}$, and the terminal behavior is observed. We fitted a relaxation time spectrum $H(\tau)$ to $G^{*}(\omega)$ by applying the nonlinear regularization routine of the software NLREG (Freiburg Materials Research Center 2001). Then, the zero shear rate viscosity $\eta_{0}=2.05 \times$ $10^{5} \mathrm{Pas}$, and the steady shear compliance $J_{e}^{0}=2.55 \times$ $10^{-5} \mathrm{~Pa}^{-1}$ at $190^{\circ} \mathrm{C}$ were determined using the relations (Ferry 1980)

$\eta_{0}=\int_{-\infty}^{\infty} \tau H(\tau) d \ln \tau$ 


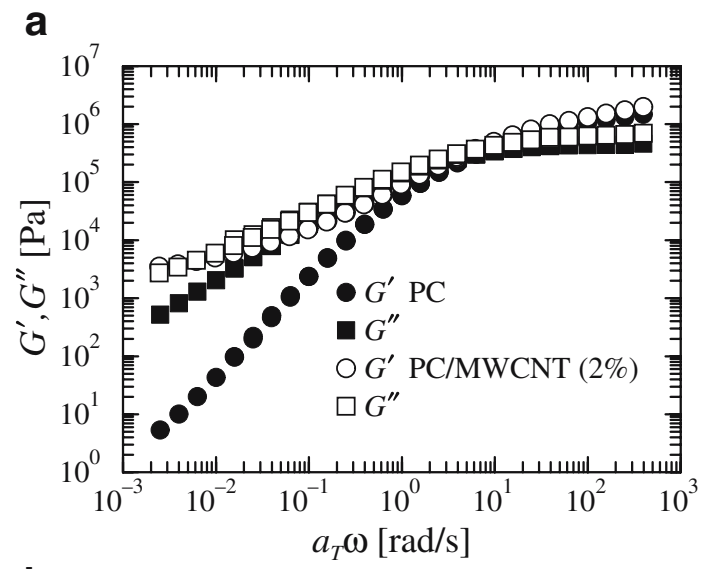

b

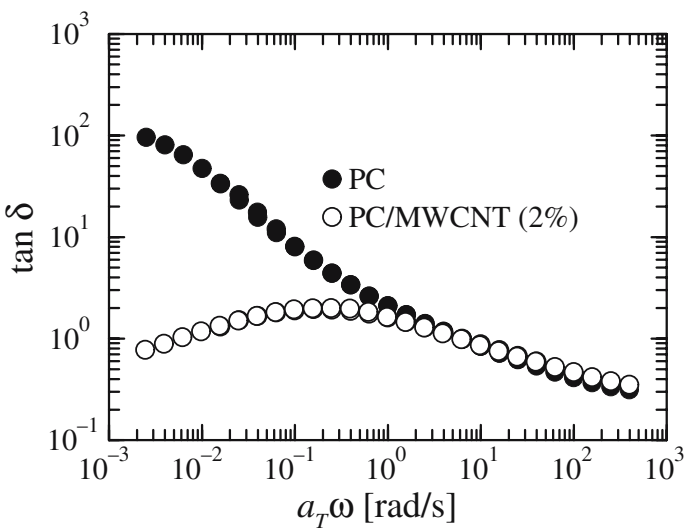

Fig. 1 Complex modulus $G^{*}=G^{\prime}+i G^{\prime \prime}$ that was shifted to the reference temperature $T_{0}=190^{\circ} \mathrm{C}$ for PC Lexan HF 1110 (filled symbols) and the PC/MWCNT ( $2 \mathrm{wt} \%$ ) composite (open symbols): a Storage modulus $G^{\prime}$ and loss modulus $G^{\prime \prime}$. b Loss tangent $\tan \delta$. The angular frequency is denoted by $\omega$ and the shift factor by $a_{T}$

and

$J_{e}^{0}=\eta_{0}^{-2} \int_{-\infty}^{\infty} \tau^{2} H(\tau) d \ln \tau$.

The average relaxation time can be estimated by $\langle\tau\rangle=$ $J_{e}^{0} \eta_{0}$ which leads to $\langle\tau\rangle=5.2 \mathrm{~s}$ for the PC melt.

Figure 1a also presents the shifted complex modulus for the composite. A detailed inspection of the data for the composite reveals that the measured data for $T=190^{\circ} \mathrm{C}$ and $T=210^{\circ} \mathrm{C}$ can be only dissatisfactorily merged. The storage modulus and the loss modulus of the PC/MWCNT ( $2 \mathrm{wt} \%)$ composite exceed the corresponding values of pure $\mathrm{PC}$ at each frequency, see Fig. 1a. For $\omega$ values that are larger than $6.2 \mathrm{rad} \mathrm{s}^{-1}$, the storage modulus $G^{\prime}$ of PC/MWCNT ( $2 \mathrm{wt} \%$ ) composite is larger than the loss modulus $G^{\prime \prime}$ of the composite. In the intermediate $\omega$ range of our shear rheometer,
$3.9 \times 10^{-3} \mathrm{rad} \mathrm{s}^{-1} \leq \omega \leq 6.2 \mathrm{rad} \mathrm{s}^{-1}$, the loss modulus $G^{\prime \prime}$ exceeds the storage modulus $G^{\prime}$. For $\omega<3.9 \times$ $10^{-3} \mathrm{rad} \mathrm{s}^{-1}$, the storage modulus is again larger than the loss modulus.

Figure $1 \mathrm{~b}$ presents the loss tangent $\tan \delta=G^{\prime \prime} / G^{\prime}$ as a function of the frequency $\omega$ at reference temperature $T_{0}=190^{\circ} \mathrm{C}$. The loss tangent $\tan \delta$ of the pure material monotonically increases with decreasing $\omega$. Such a behavior is commonly observed for melts of homopolymers. In contrast, the loss tangent of the PC/MWCNT ( $2 \mathrm{wt} \%$ ) composite displays a pronounced maximum at $\omega \approx 0.25 \mathrm{rad} \mathrm{s}^{-1}$. For smaller and larger $\omega$ values, the loss factor $\tan \delta$ decreases. At large frequencies, the loss tangent of the PC/MWCNT composite only slightly exceeds the loss tangent of pure PC.

In Fig. 2, we plot the ratios

$r_{1}(\omega)=G_{\mathrm{PC} / \operatorname{MWCNT}(2 \%)}^{\prime}(\omega) / G_{\mathrm{PC}}^{\prime}(\omega)$

$r_{2}(\omega)=G_{\mathrm{PC} / \operatorname{MWCNT}(2 \%)}^{\prime \prime}(\omega) / G_{\mathrm{PC}}^{\prime \prime}(\omega)$,

to demonstrate the difference in the linear viscoelastic properties of pure PC and the PC/MWCNT composite. At large frequencies, $r_{1}$ and $r_{2}$ attain roughly constant values, and the storage and the loss modulus of the composite are simply shifted to higher values. The shift factors range between 1.1 and 1.5. These remarkably large shift factors for such a low content of fillers are caused by the high aspect ratio of the carbon nanotubes that leads to a network-like arrangement. At low frequencies, the values of $r_{1}$ and $r_{2}$ are much larger than unity, which indicates the crossover to the regime where the influence of the carbon nanotubes is strongly pronounced.

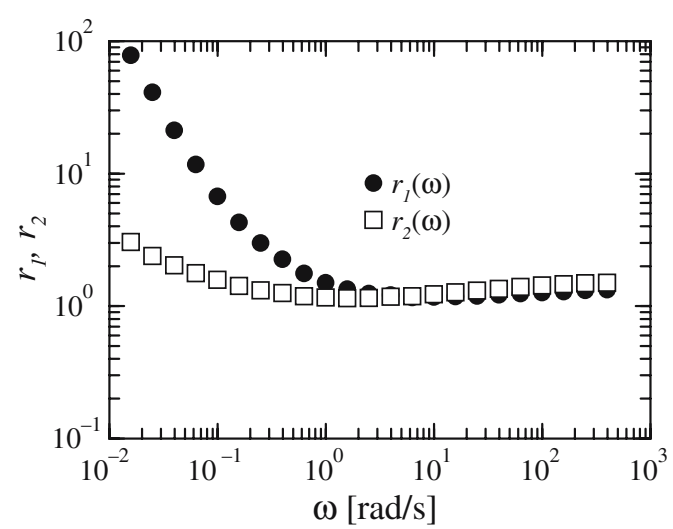

Fig. 2 The ratios $r_{1}(\omega)$ and $r_{2}(\omega)$ of the storage and the loss modulus of the PC/MWCNT ( $2 \mathrm{wt} \%$ ) composite and pure $\mathrm{PC}$ as a function of $\omega$ at temperature $T=190^{\circ} \mathrm{C}$, see Eqs. 3 and 4 
Elongational tests

Using the RME, we determined the transient elongational viscosity

$\mu(t)=\sigma_{\text {tensile }}(t) / \dot{\epsilon}_{0, \text { true }}$

of pure $\mathrm{PC}$ and the PC/MWCNT composite ( $2 \mathrm{wt} \%$ ) for the four selected Hencky strain rates. In Eq. 5, $\sigma_{\text {tensile }}$ denotes the measured tensile stress, and $\dot{\epsilon}_{0 \text {,true }}$ is the true Hencky strain rate that was determined after the measurements. The transient elongational viscosity $\mu(t)$ of pure $\mathrm{PC}$ and the PC/MWCNT composite is shown in Fig. 3. In addition, we plot the linear viscoelastic prediction $\mu^{\mathrm{o}}(t)$ for $\mu(t)$ of pure PC, i.e., $\mu^{\mathrm{o}}(t)=3 \eta^{\circ}(t)$ with the transient linear shear viscosity $\eta^{\circ}(t)$ (Ferry 1980)

$\eta^{\mathrm{o}}(t)=\int_{-\infty}^{\infty} \tau H(\tau)[1-\exp (-t / \tau)] d \ln \tau$.

The elongational viscosity of pure PC monotonically increases with time $t$. At large times, the elongational viscosity attains a stationary value for $\dot{\epsilon}_{0}=0.1 \mathrm{~s}^{-1}$. For $\dot{\epsilon}_{0} \geq 0.3 \mathrm{~s}^{-1}, \mu(t)$ slightly increases with strain rate at large $t$. Our data reveal that the transient elongational viscosity $\mu(t)$ of pure PC for $\dot{\epsilon}_{0}=0.1 \mathrm{~s}$ is shifted by a factor of approximately 1.1 compared to the linear viscoelastic prediction $\mu^{\mathrm{o}}(t)$. This shift in viscosity is most probably caused by a small temperature difference between the temperature $T_{1}$ of the shear rheometer and the temperature $T_{2}$ of the elongational rheometer, which can be estimated by $\Delta T=T_{2}-T_{1}=-0.35^{\circ} \mathrm{C}$ using the Williams-Landel-Ferry equation.

The elongational viscosity of the PC/MWCNT composite also increases with time. For $\dot{\epsilon}_{0}=0.1 \mathrm{~s}^{-1}$, the elongational viscosity $\mu(t)$ of the composite is shifted by a factor of approximately 1.15 to $\mu(t)$ of pure PC, but the overall shape of the transient elongational viscosity for pure PC and the composite are very similar for $\dot{\epsilon}_{0}=0.1 \mathrm{~s}^{-1}$. For larger strain rates, the elongational viscosity of the PC/MWCNT composite exceeds the $\mu$ values of pure $\mathrm{PC}$ at small times. At larger times, the elongational viscosity of the composite is smaller than $\mu(t)$ of pure PC. This trend is more pronounced with increasing strain rate.

Fig. 3 Transient elongational viscosity $\mu$ as a function of time $t$ of pure $\mathrm{PC}$ and the PC/MWCNT $\left(2 \mathrm{wt} \%\right.$ ) composite at $T=190^{\circ} \mathrm{C}$. We have also plotted the linear viscoelastic elongational viscosity $\mu^{\mathrm{o}}(t)=3 \eta^{\mathrm{o}}(t)$ for pure PC. The Hencky strain rate $\dot{\epsilon}_{0}$ is a $\dot{\epsilon}_{0}=$ $0.1 \mathrm{~s}^{-1}, \mathbf{b} \dot{\epsilon}_{0}=0.3 \mathrm{~s}^{-1}, \mathbf{c} \dot{\epsilon}_{0}=0.7 \mathrm{~s}^{-1}$, and $\mathbf{d} \dot{\epsilon}_{0}=1.0 \mathrm{~s}^{-1}$ a

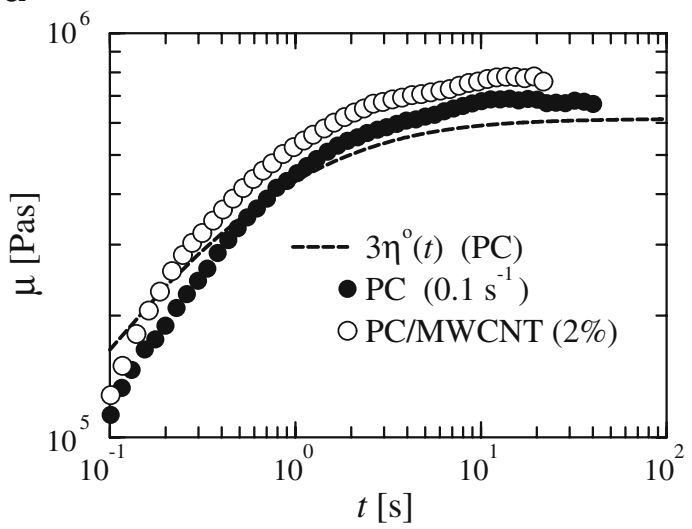

b

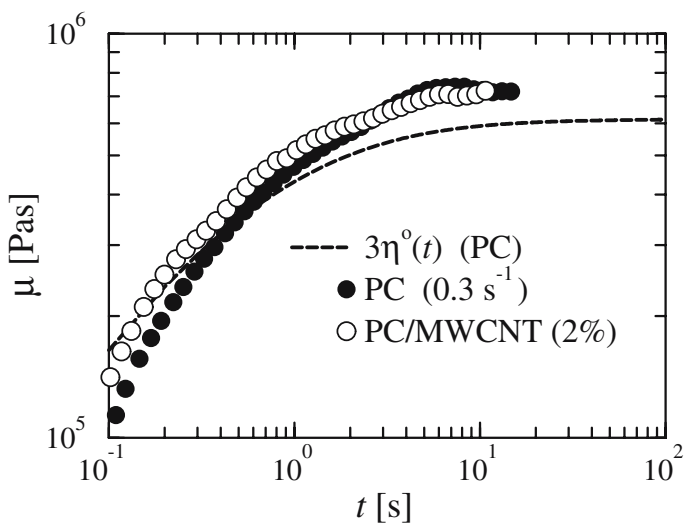

C

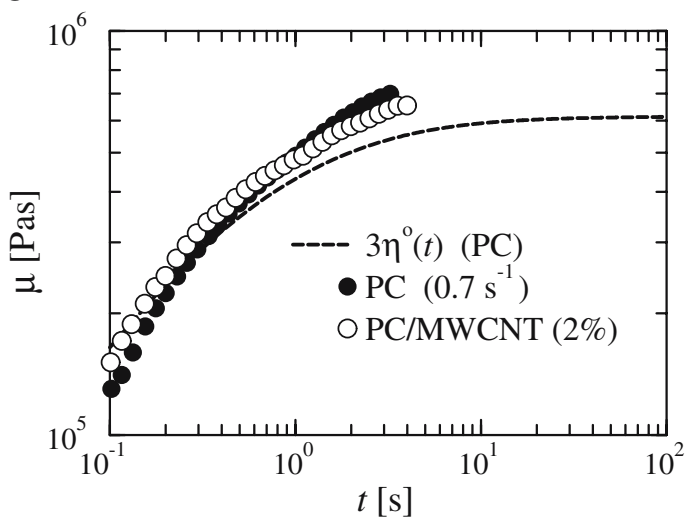

d

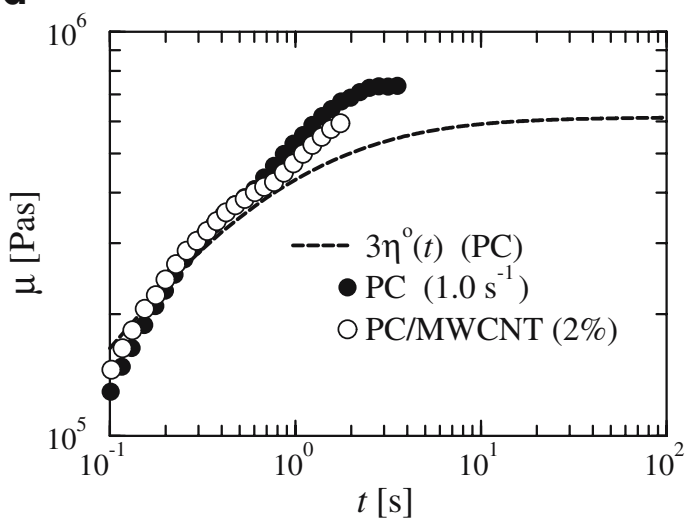


The transient recovery can be quantified by the timedependent recovered stretch $\lambda_{r}$, which is given by

$\lambda_{r}\left(t^{\prime}\right)=L_{\max } / L\left(t^{\prime}\right)$.

Here, $L_{\max }$ is the length of the sample at cutting time $t=t_{\max }$, and $L\left(t^{\prime}\right)$ denotes the length of the sample at recovery time $t^{\prime}=t-t_{\max }$. The results for the transient recovered stretch $\lambda_{r}$ of PC and the PC/MWCNT composite are shown in Figs. 4 and 5 for the four selected Hencky strain rates.

The test temperature was $T=190{ }^{\circ} \mathrm{C}$, and the maximum Hencky strain $\epsilon_{\max }=2.4$. The data reveal that the recovered stretch increases with increasing strain rate both for pure PC and the PC/MWCNT ( $2 \mathrm{wt} \%$ ) composite (Fig. 4). In the initial stage of recovery, the overall shape of the $\lambda_{r}\left(t^{\prime}\right)$ curves are very similar for pure PC and the PC/MWCNT (2 wt \%) composite, see Fig. $5 \mathrm{a}$ and $\mathrm{b}$. For both pure PC and the composite, the

a

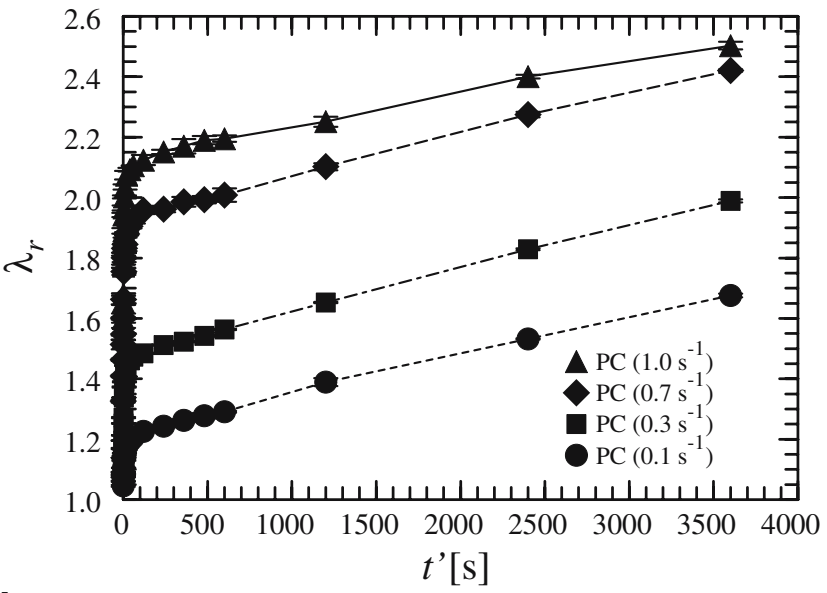

b

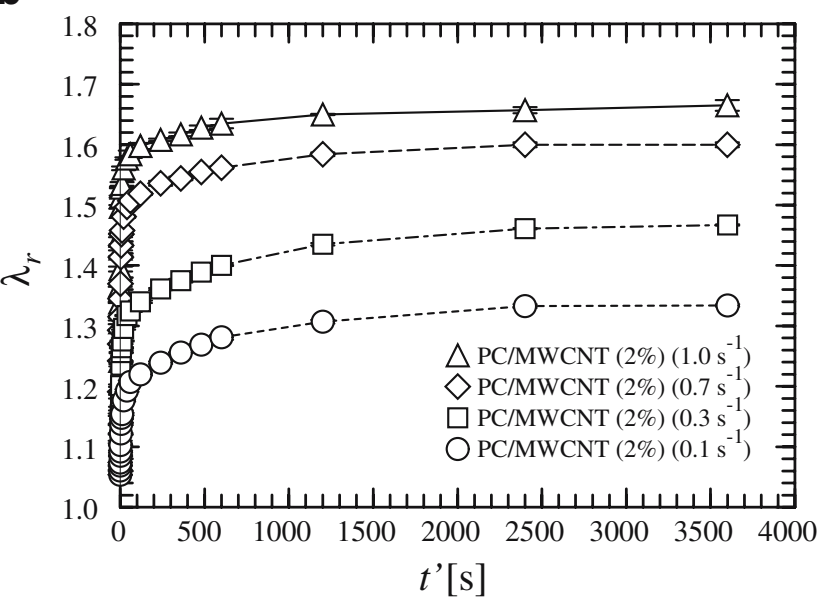

Fig. 4 Time-dependent recovered stretch $\lambda_{r}$ as a function of recovery time $t^{\prime}$ for a $\mathrm{PC}$ and $\mathbf{b}$ the PC/MWCNT ( $2 \mathrm{wt} \%$ ) composite. The test temperature was $T=190{ }^{\circ} \mathrm{C}$, and the maximum Hencky strain $\epsilon_{\max }=2.4$. The Hencky strain rate is indicated

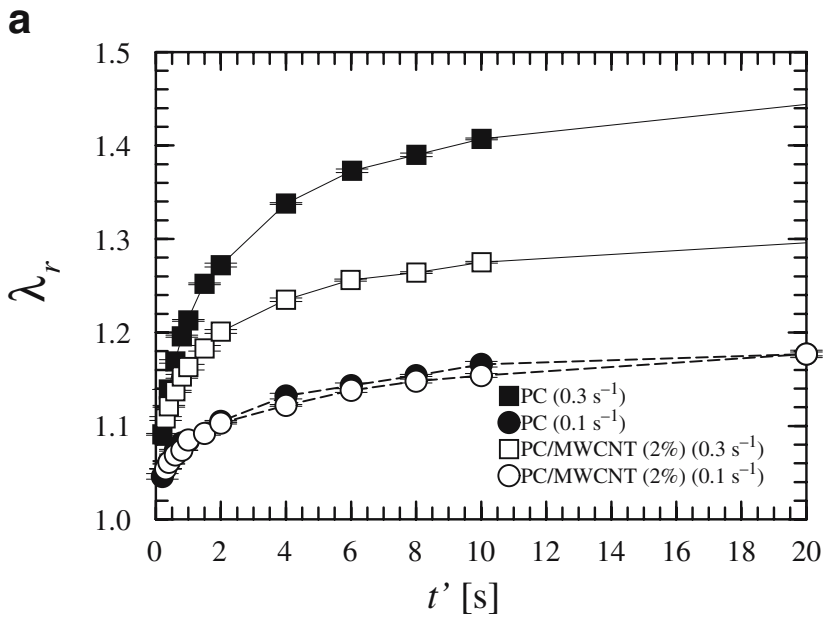

b

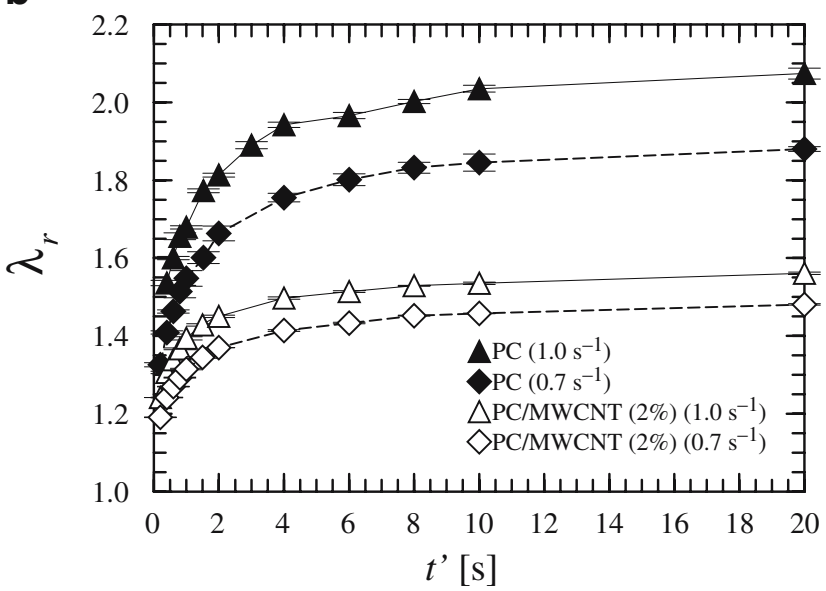

Fig. 5 Comparison of the $\lambda_{r}\left(t^{\prime}\right)$ values of $\mathrm{PC}$ and the PC/MWCNT $(2 \mathrm{wt} \%)$ composite for $t^{\prime} \leq 20 \mathrm{~s}$. The data are the same as in Fig. 4. The Hencky strain rate is a $\dot{\epsilon}_{0}=0.1 \mathrm{~s}^{-1}$ and $\dot{\epsilon}_{0}=0.3 \mathrm{~s}^{-1} . \mathbf{b} \dot{\epsilon}_{0}=0.7 \mathrm{~s}^{-1}$ and $\dot{\epsilon}_{0}=1.0 \mathrm{~s}^{-1}$

recovered stretch $\lambda_{r}$ increases rapidly after the sample has been cut. The recovered stretch of the composite nearly equals the corresponding value of pure $\mathrm{PC}$ for $\dot{\epsilon}_{0}=0.1 \mathrm{~s}^{-1}$, but is much smaller than $\lambda_{r}$ of pure PC for $\dot{\epsilon}_{0} \geq 0.3 \mathrm{~s}^{-1}$. At large recovery times, the pure material and the composite display a different behavior: At large $t^{\prime}$, the transient recovered stretch of pure PC still increases, but the slope of the $\lambda_{r}\left(t^{\prime}\right)$ curve is much smaller than the slope in the initial stage, cf. Fig. 4a. In the case of the composite, the transient recovered stretch $\lambda_{r}$ also increases for $20 \mathrm{~s} \leq t^{\prime} \leq 1000 \mathrm{~s}$, i.e., for recovery times that are significantly larger than the largest retardation times of the macromolecules (Fig. 4b). However, at recovery times that are larger than approximately $1,000 \mathrm{~s}$, the recovered stretch of the composite almost ceases.

\section{Morphology}

Figure 6 shows a thin section of the compressionmolded PC/MWCNT ( $2 \mathrm{wt} \%$ ) composite before elon- 


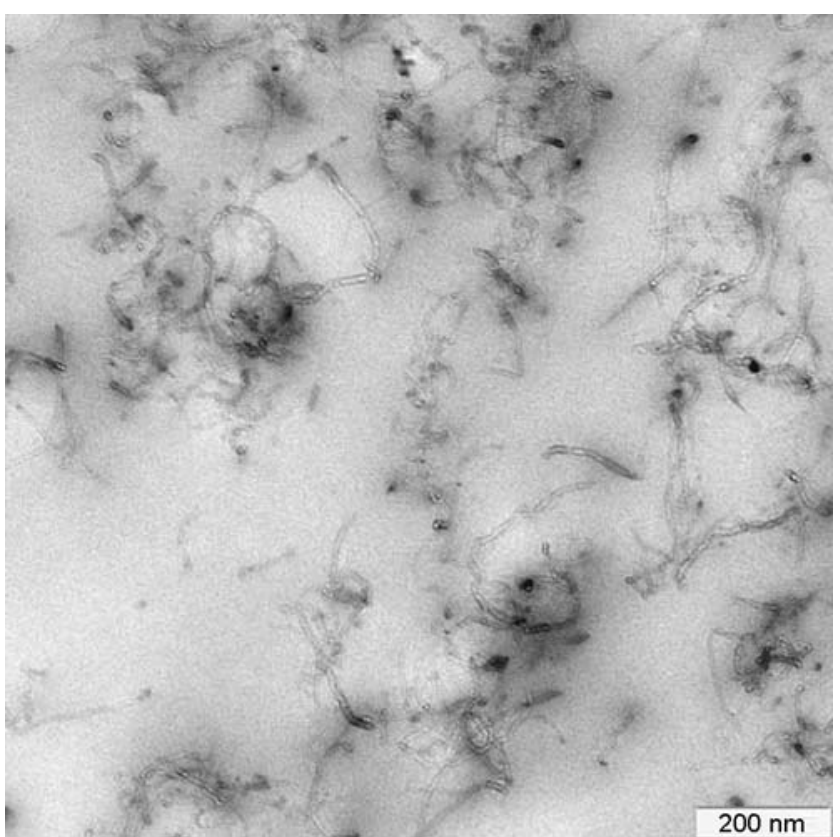

Fig. 6 TEM of the PC/MWCNT (2wt\%) composite before elongation

gation. The MWCNT of this study were not completely straight but partially bent. The deviations from the linear shape are assumed to result from the formation of defects during the CVD synthesis of the nanotubes. No preferential orientation of the nanotubes is visible, which indicates that the preparation of the thin slices with an ultramicrotome did not orient the nanotubes. As the weight fraction of the carbon nanotubes in the composite was above the percolation threshold for electrical conductivity (Satapathy et al. 2007), the carbon nanotubes were far away from being isolated and formed a network-like arrangement. This arrangement consisted of regions with higher and smaller density of carbon nanotubes, i.e., "clusters" of randomly entangled carbon nanotubes and regions of isolated carbon nanotubes, see Fig. 6. The "clusters" are uniformly distributed in the composite. The overall dispersion of the nanotubes is comparable to other composites with a highly viscous matrix, cf. Andrews et al. (2002), Sennett et al. (2003), and Kim and Kim (2006). Generally, the dispersion of carbon nanotubes in a highly viscous polymer matrix is much more difficult than in a low viscous matrix. Aggregation of nanotubes is possibly increased during compression molding (which is necessary to obtain relaxed, stress-free samples for

Fig. 7 TEM of the elongated $\left[\epsilon_{s}=\epsilon_{\max }=2.4\right.$, (a and $\left.\left.\mathbf{b}\right)\right]$ and recovered $\left[t^{\prime}=60 \mathrm{~min},(\mathbf{c})\right] \mathrm{PC} / \mathrm{MWCNT}(2 \mathrm{wt} \%)$ composite. The white bar indicates the direction of stretching. The test parameters were $\dot{\epsilon}_{0}=0.3 \mathrm{~s}^{-1}, \epsilon_{\max }=2.4$ and $T=190^{\circ} \mathrm{C}$
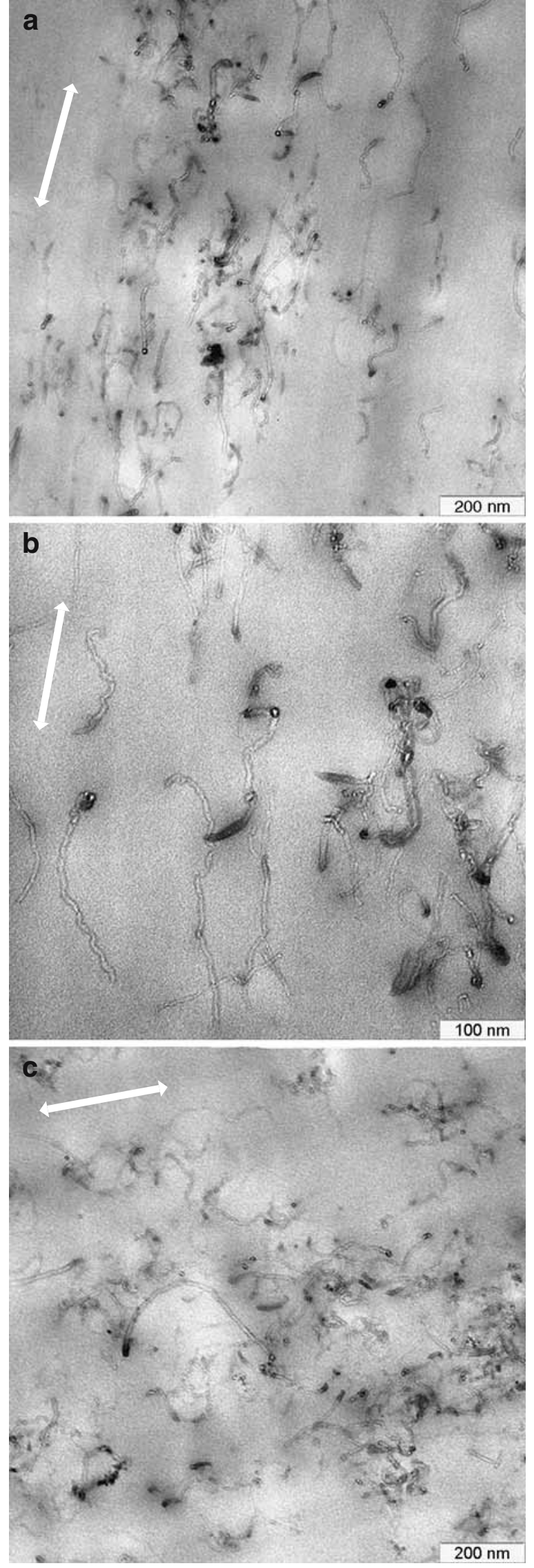
elongational rheometry) and can influence the electrical conductivity and the viscosity (Bryning et al. 2005; Xu et al. 2005).

The influence of macroscopic melt elongation with Hencky strain rate $\dot{\epsilon}_{0}=0.3 \mathrm{~s}^{-1}$ is shown in Fig. 7. After elongation to the macroscopic Hencky strain $\epsilon_{s}=$ $\epsilon_{\max }=2.4$, the isolated carbon nanotubes were oriented parallel to the flow direction. Figure $7 \mathrm{a}$ and $\mathrm{b}$ indicate that the nanotubes are partially straightened. In addition, the "clusters" with higher density of intertwined carbon nanotubes were oriented by the elongational flow, whereas the random arrangement within them still persisted. Some "clusters" were partially dissolved during elongation. Such microstructural breakup phenomena also appear during shear of polystyrene/carbon nanofiber melts (Wang et al. 2006). At recovery time $t^{\prime}=60 \mathrm{~min}$, the carbon nanotubes were not aligned anymore but randomly oriented, see Fig. 7c.

\section{Discussion}

The loss tangent (Fig. 1b) of the PC/MWCNT (2 wt \%) composite reveals that, for very small and very large $\omega$, a larger amount of elastic energy is stored in the system than energy is dissipated. At large frequencies, the stress in the PC matrix is much larger than the stresses that are caused by the interactions between the carbon nanotubes and the polymer-carbon nanotube interactions. Therefore, the loss tangent of pure $\mathrm{PC}$ and the composite do not differ much and are smaller than unity at large $\omega$. At low frequencies, the stress in the PC matrix is very small, as $G^{\prime}$ and $G^{\prime \prime}$ attain very small values. For low $\omega$, the stresses resulting from the arrangement of carbon nanotubes and the stresses between carbon nanotubes and macromolecules exceed the stress in the PC matrix. Elastic interactions that result from the carbon nanotubes arrangement are physical debonding between the nanotubes and bending of the nanotubes. Because of the dense networklike arrangement and the resulting elastic interactions (physical debonding and bending), the storage modulus is increased at low frequencies. In the low frequency range, the nanocomposite behaves like a solid. A plateau that is independent of $\omega$ occurs at very low frequencies that are smaller than the accessible frequency range of our shear rheometer, see also Pötschke et al. (2004) and Zhang et al. (2006a). This low frequency behavior was also observed for other composites, e.g., melts of polystyrene/carbon nanofiber composites as shown by Wang et al. (2006). The crossover from the polymer melt-like behavior at large $\omega$ to the carbon nanotubes dominated regime at low $\omega$ is roughly given by $\omega \approx 1 \mathrm{rads}^{-1}$. In the intermediate range between low and large frequencies, the loss tangent $\tan \delta$ depicts a maximum because of the interplay of the stress in the PC matrix and the stress resulting from the carbon nanotubes: The polymer matrix contributes to an increasing $\tan \delta$ and the carbon nanotubes to a decreasing $\tan \delta$ for decreasing $\omega$. The competition of these two contributions results in a maximum for the loss tangent.

The failure of the time-temperature superposition for the composite hints that the temperature dependence of the relevant relaxation processes are different in the pure polymer and in the composite. The interactions resulting from the carbon nanotubes are less influenced by the temperature than the entropy elasticity of the polymer melt. Consequently, the relaxation times in the composite that are associated with the carbon nanotubes arrangement depend differently on the temperature when compared with the relaxation times of the PC melt.

The comparison of the transient elongational viscosity $\mu$ of pure $\mathrm{PC}$ and the composite reveals that the addition of $2 \mathrm{wt} \%$ MWCNT only moderately changes the value and the time dependence of the elongational viscosity, see Fig. 3. In melt elongation, the stress of the PC matrix is much higher than the stress that is caused by the carbon nanotubes. Only small stresses are necessary to deform the network-like arrangement of carbon nanotubes. The behavior in elongation parallels the high frequency behavior of the composite where the polymer matrix mostly contributed to the complex modulus.

Figure 4 shows that the transient recovered stretch $\lambda_{r}$ of the PC melt is composed of two contributions with clearly separated time scales, i.e., the molecular driven recovery and the surface tension driven recovery portions. In the initial stage of recovery, the stretched macromolecules recover to an isotropic coiled state. The time scale of the recovery of the macromolecules is comparable to the average relaxation time. At approximately $t^{\prime}=20 \mathrm{~s}$, a crossover takes place. At recovery times that are larger than $20 \mathrm{~s}$, only the surface tension between the sample and the nitrogen atmosphere contributes to the recovery of the melt. This is indicated by a smaller value of the slope of the $\lambda_{r}\left(t^{\prime}\right)$ curve, which can be clearly seen in Fig. 4a.

In Fig. 5, the recovered stretch of pure $\mathrm{PC}$ and the composite for the same Hencky strain rate are compared. The analysis of these data reveals that the average retardation time of the macromolecules is not significantly changed by the addition of carbon nanotubes. For $\dot{\epsilon}_{0}=0.1 \mathrm{~s}^{-1}$, the recovered stretch of pure $\mathrm{PC}$ and the PC/MWCNT composite does not differ much, which indicates that the recovery of only slightly 
stretched macromolecules is not hindered by the carbon nanotubes. However, for $\dot{\epsilon}_{0} \geq 0.3 \mathrm{~s}^{-1}$, the $\lambda_{r}$ values at the same recovery time $t^{\prime}$ are enormously reduced. We interpreted this result by taking into account the influence of the carbon nanotubes on large deformations of the macromolecules: During elongation, the macromolecules are less stretched in presence of the carbon nanotubes because the macromolecules partially stick to the nanotubes and no slip occurs between the nanotubes and the surrounding matrix. Consequently, the flow field consists of shear components in addition to the elongational components. Generally, in shear, the polymers are less stretched than in elongation, which lowers the amount of reversible deformations and, thus, leads to a smaller recovered stretch. At large recovery times, $\lambda_{r}$ increases more rapidly for pure PC than for the composite. Whereas pure PC does not stop to recover during the time of our measurements, the transient recovered stretch of the nanocomposite increases only slowly for $20 \mathrm{~s} \leq t^{\prime} \leq 1,000 \mathrm{~s}$ and attains an almost stationary value at the end of our experiments. These data give a hint that the carbon nanotubes network can still be slightly deformed after the molecular recovery has been terminated, i.e., for $20 \mathrm{~s} \leq t^{\prime} \leq 1,000 \mathrm{~s}$. At recovery times $t^{\prime} \geq 1,000 \mathrm{~s}$, the yield stress of the composite prohibits further flow of the nanocomposites. The yield stress is caused by the elastic network of carbon nanotubes, similar to the polystyrene/carbon nanofiber composites of Wang et al. 2006. Small stress can only marginally deform this network and cannot create a permanent flow. As the stress that is caused by the surface tension is too small, the surface tension driven recovery portion is enormously reduced.

\section{Conclusions}

In this work, we investigated the linear viscoelastic shear and the elongational properties of a PC/MWCNT ( $2 \mathrm{wt} \%$ ) composite in the melt. The rheological experiments revealed that the influence of the carbon nanotubes was large when the externally applied stress was small, i.e., in the low frequency range of the harmonic shear oscillations and in recovery. In melt elongation, the stress in the polymer matrix was much larger than the stresses caused by the carbon nanotubes, and thus, the transient elongational viscosity was not much influenced by the addition of $2 \mathrm{wt} \%$ carbon nanotubes. In recovery after melt elongation, the average retardation time of the macromolecules was not significantly changed by the presence of the nanotubes. Our recovery data indicate that the arrangement of carbon nanotubes led to a yield stress and prohibited large extensions of the macromolecules during elongation. TEM showed that the carbon nanotubes were randomly oriented and partially intertwined in regions with larger density of nanotubes ("clusters") before elongation. These clusters were uniformly distributed in the composite. In addition, individual carbon nanotubes were present between the clusters. In melt elongation, the isolated carbon nanotubes were aligned. At large recovery times, the carbon nanotubes were again randomly oriented.

Acknowledgements The authors thank Polymer Service GmbH (Merseburg, Germany) for the TEM investigations and Professors Chr. Friedrich, J. Meissner, H.C. Öttinger, and M. Wagner for very valuable discussions. The continuous support of J. Hostettler, F. Mettler, and W. Schmidheiny is gratefully acknowledged. We also thank $\mathrm{H}$. Kunath for the extraction experiments, P. Treppe, and D. Voigt for the gel permeation chromatography analysis, Dr. J. Pionteck for the density measurements, and K. Arnhold and L. Häußler for the differential scanning calorimetry investigations.

\section{References}

Andrews R, Jacques D, Minot M, Rantell T (2002) Fabrication of carbon multiwall nanotube/polymer composites by shear mixing. Macromol Mater Eng 287:395-403

Breuer O, Sundararaj U (2004) Big returns from small fibers: a review of polymer/carbon nanotubes composites. Polym Compos 25:630-645

Bryning MB, Islam MF, Kikkawa JM, Yodh AG (2005) Very low conductivity threshold in bulk isotropy single-walled carbon nanotube-epoxy composites. Adv Mater 17:1186-1191

Camponeschi E, Florkowski B, Vance R, Garrett G, Garmestani H, Tannenbaum R (2006) Uniform directional alignment of single-walled carbon nanotubes in viscous polymer flow. Langmuir 22:1858-1862

Coleman JN, Khan U, Gunko YK (2006) Mechanical reinforcement of polymers using carbon nanotubes. Adv Mater 18:689-706

Dai H, Hafner JH, Rinzler AG, Colbert DT, Smalley RE (1996) Nanotubes as nanoprobes in scanning probe microscopy. Nature 384:147-150

Du F, Scogna RC, Zhou W, Brand S, Fischer JE, Winey KI (2004) Nanotube networks in polymer nanocomposites: rheology and electrical conductivity. Macromolecules 37:9048-9055

Ferry, JD (1980) Viscoelastic properties of polymers (3rd edn). Wiley, New York

Freiburg Materials Research Center (Service Group Scientific Data Processing) (2001) NLREG (non-linear-regularization), Software Version Rheology 2.2, August 2001

Fry D, Langhorst B, Kim H, Grulke E, Wang H, Hobbie EK (2005) Anisotropy of sheared carbon-nanotube suspensions. Phys Rev Lett 95:038304-1-038304-4

Handge UA, Pötschke P $(2004,2005)$ Interplay of rheology and morphology in melt elongation and subsequent recovery of polystyrene/poly(methyl methacrylate) blends. J Rheol 48:1103-1122, erratum in J Rheol 49:1553 
Hobbie EK, Wang H, Kim H, Lin-Gibson S, Grulke EA (2003) Orientation of carbon nanotubes in a sheared polymer melt. Phys Fluids 15:1196-1202

Hough LA, Islam MF, Janmey PA, Yodh AG (2004) Viscoelasticity of single wall carbon nanotube suspensions. Phys Rev Lett 93:168102-1-168102-4

Huang YY, Ahir SV, Terentjev EM (2006) Dispersion rheology of carbon nanotubes in a polymer matrix. Phys Rev B 73:125422-1-125422-9

Kim JY, Kim SH (2006) Influence of multiwall carbon nanotube on physical properties of poly(ethylene 2,6naphthalate) nanocomposites. J Polym Sci B Polym Phys 44: 1062-1071

Larson RG (1999) The structure and rheology of complex fluids. Oxford Univ. Press, New York, p 286

Lee SB, Teo KBK, Chhowalla M, Hasko DG, Amaratunga GAJ, Milne WI, Ahmed H (2002) Study of multi-walled carbon nanotube structures fabricated by PMMA suspended dispersion. Microelectron Eng 61-62:475-483

Lin B, Sundararaj U, Pötschke P (2006) Melt mixing of polycarbonate with multi-walled carbon nanotubes in miniature mixers. Macromol Mater Eng 291:227-238

Lin-Gibson S, Pathak JA, Grulke EA, Wang H, Hobbie EK (2004) Elastic flow instability in nanotube suspensions. Phys Rev Lett 92:048302-1-048302-4

Martin CA, Sandler JKW, Shaffer MSP, Schwarz MK, Bauhofer W, Schulte K, Windle AH (2004) Formation of percolating networks in multi-wall carbon-nanotube-epoxy composites. Compos Sci Technol 64:2309-2316

McNally T, Pötschke P, Halley P, Murphy M, Martin D, Bell SEJ, Brennan GP, Bein D, Lemoine P, Quinn JP (2005) Polyethylene multiwalled carbon nanotube composites. Polymer 46:8222-8232

Meincke O, Kaempfer D, Weickmann H, Friedrich C, Vathauer M, Warth H (2004) Mechanical properties and electrical conductivity of carbon-nanotube filled polyamide- 6 and its blends with acrylonitrile/butadiene/styrene. Polymer 45: 739-748

Meissner J, Hostettler J (1994) A new elongational rheometer for polymer melts and other highly viscoelastic liquids. Rheol Acta 33:1-21

Mitchell CA, Bahr JL, Arepalli S, Tour JM, Krishnamoorti R (2002) Dispersion of functionalized carbon nanotubes in polystyrene. Macromolecules 35:8825-8830

Ooi YW, Sridhar T (2004) Resistance to uniaxial extensional flow of fibre suspensions. Rheol Acta 43:223-231

Pötschke P, Fornes TD, Paul DR (2002) Rheological behavior of multiwalled carbon nanotube/polycarbonate composites. Polymer 43:3247-3255
Pötschke P, Bhattacharyya AR, Janke A, Goering H (2003) Melt mixing of polycarbonate/multi-wall carbon nanotubes composites. Compos Interfaces 10:389-404

Pötschke P, Abdel-Goad M, Alig I, Dudkin S, Lellinger D (2004) Rheological and dielectrical characterization of melt mixed polycarbonate-multiwalled carbon nanotube composites. Polymer 45:8863-8870

Pötschke P, Brünig H, Janke A, Fischer D, Jehnichen D (2005) Orientation of multiwalled carbon nanotubes in composites with polycarbonate by melt spinning. Polymer 46: 10355-10363

Satapathy BK, Weidisch R, Pötschke P, Janke A (2007) Tough-to-brittle transition in multiwalled carbon nanotube (MWNT)/polycarbonate nanocomposites. Compos Sci Technol 67:867-879

Schmid CF, Switzer LH, Klingenberg DJ (2000) Simulations of fiber flocculations: effects of fiber properties and interfiber friction. J Rheol 44:781-809

Schweizer T (2000) The uniaxial elongational rheometer RMEsix years of experience. Rheol Acta 39:428-443

Sennett M, Welsh E, Wright JB, Li WZ, Wen JG, Ren ZF (2003) Dispersion and alignment of carbon nanotubes in polycarbonate. Appl Phys A 76:111-113

Seo MK, Park SJ (2004) Electrical resistivity and rheological behaviors of carbon nanotubes-filled polypropylene composites. Chem Phys Lett 395:44-48

Start PR, Hudson SD, Hobbie EK, Migler KB (2006) Breakup of carbon nanotube flocs in microfluidic traps. J Colloid Interface Sci 297:631-636

Subramoney S (1998) Novel nanocarbons-structure, properties, and potential applications. Adv Mater 10:1157-1171

Switzer III LH, Klingenberg DJ (2003) Rheology of sheared flexible fiber suspensions via fiber-level simulations. J Rheol 47:759-778

Trevelyan BJ, Mason SG (1951) Particle motions in sheared suspensions. I. Rotations. J Colloid Sci 6:354-367

Wang Y, Xu J, Bechtel SE, Koelling KW (2006) Melt shear rheology of carbon nanofiber/polystyrene composites. Rheol Acta 45:919-941

Xu J, Chatterjee S, Koelling KW, Wang Y, Bechtel SE (2005) Shear and extensional rheology of carbon nanofiber suspensions. Rheol Acta 44:537-562

Zhang Q, Lippits DR, Rastogi S (2006a) Dispersion and rheological aspects of SWNTs in ultrahigh molecular weight polyethylene. Macromolecules 39:658-666

Zhang Q, Rastogi S, Chen D, Lippits D, Lemstra PJ (2006b) Low percolation threshold in single-walled carbon nanotube/high density polyethylene composites prepared by melt processing technique. Carbon 44:778-785 